\title{
Improved Myocardial Scar Characterization by Super-Resolution Reconstruction in Late Gadolinium Enhanced MRI`
}

Oleh Dzyubachyk ${ }^{1, \star \star}$, Qian Tao ${ }^{1, \star \star}$, Dirk H. J. Poot ${ }^{3}$, Hildo Lamb ${ }^{1}$, Katja Zeppenfeld ${ }^{2}$, Boudewijn P. F. Lelieveldt ${ }^{1,4}$, and Rob J. van der Geest ${ }^{1}$

1 Department of Radiology, Leiden University Medical Center, Leiden, The Netherlands

2 Department of Cardiology, Leiden University Medical Center, Leiden, The Netherlands

3 Departments of Radiology and Medical Informatics, Erasmus MC - University

Medical Center Rotterdam, Rotterdam, The Netherlands

4 Intelligent Systems Department, Delft University of Technology,

Delft, The Netherlands

\begin{abstract}
Image resolution is an important factor for accurate myocardial scar assessment from late gadolinium enhanced (LGE) MR. It has been shown that the conventionally used short-axis (SA) LGE acquisition with anisotropic resolution may overestimate the scar size due to partial volume effect, undermining the prognostic and diagnostic accuracy of LGE MRI in critical clinical applications. In this work, we present a method for combining three complementary anisotropic orthogonal LGE sequences of the heart region into a single isotropic volume. Our algorithm is based on the super-resolution reconstruction technique and employs joint localized gradient-correlation-based technique for compensation of breathing motion. The proposed method was validated on the gold standard electroanatomical voltage mapping (EAVM) data of 15 post-infarction patients. The reconstructed myocardial scar image demonstrated improved agreement with the EAVM compared to the conventional SA image, especially at the clinically significant gray zone region.
\end{abstract}

Keywords: Myocardial scar, late gadolinium enhanced MR, image registration, super-resolution reconstruction.

\section{Introduction}

Myocardial infarction occurs in more than 7 million patients worldwide annually, and is a frequent cause for cardiac arrhythmia and sudden cardiac death. Accurate characterization of the post-infarction myocardial scar has important

\footnotetext{
* This research was supported by the Dutch Technology Foundation STW (Stichting Technische Wetenschappen) via grants 10894 and MEDIATE.

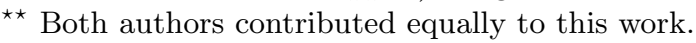


diagnostic and prognostic value for patient management and treatment [1, in e.g. defibrillator implantation and catheter ablation. In recent years, late gadolinium enhanced (LGE) MR has become the gold standard for myocardial scar imaging in clinical practice 2. In particular, the size of partially infarcted myocardial tissue, named as gray zone, has shown to be an important predictor for risks of death and arrhythmia, outperforming traditional function parameters [1].

However, recent animal studies demonstrated that the LGE-derived myocardial scar size, especially that of the gray zone, is susceptible to partial volume effect (PVE) and changes with MR imaging resolution [3. These studies underscore the significant importance of image resolution for reliable clinical evaluation of myocardial scar. Unfortunately, current clinical LGE typically has poor through-plane resolution (between 5 to $10 \mathrm{~mm}$ ) compared to in-plane resolution of about $1.5 \mathrm{~mm}$, resulting in significant PVE. To alleviate the problem, standard MR protocols often include three LGE acquisitions in orthogona 11 views: shortaxis $(\mathrm{SA})$, two-chamber $(2 \mathrm{CH})$, and four-chamber $(4 \mathrm{CH})$. To evaluate each view separately is however cumbersome and suboptimal with PVE present in different directions. The newly developed three-dimensional LGE MR technique can be used for isotropic myocardial scar imaging, however, the sequence is still of limited availability and the image quality is currently compromised by motion artifacts [4].

\section{$1.1 \quad$ Related Work}

Super-resolution reconstruction (SRR) comprises a group of methods aiming at creating a high-resolution image from multiple low-resolution views of the same scene [5]. During last years, reconstruction of isotropic three-dimensional (3D) MR volumes from a set of anisotropic images has become, probably, the largest consumer of SRR techniques. This is primarily caused by intrinsic limitations imposed on the slice thickness by some MR scanning protocols. Reconstruction of the isotropic volume from three orthogonal views was, in particular, applied to cardiac MR images by using two orthogonal views [6]. However, combining all three views was concluded as not providing any additional benefit compared to averaging due to difficulty in aligning the data.

\subsection{Our Contribution}

Here we present our algorithm for reconstruction of an isotropic 3D myocardial scar volume from three orthogonal views in LGE MR sequences. To our knowledge, this is the first work to address the issue of low clinical resolution problem in MRI myocardial scar imaging, with validation on in vivo clinical measurements. Three orthogonal views are combined by the SRR framework. For correction of the heart position displacements as result of the breath-hold variations, we suggest using a localized gradient-correlation-based registration

\footnotetext{
${ }^{1}$ Here and further we refer by word "orthogonal" to both orthogonal as well as nearlyorthogonal cases.
} 
algorithm in combination with sub-voxel refinement that is performed simultaneously with the SRR. Estimation of the displacement is performed in a joint manner, using all the available image stacks simultaneously, which guarantees high quality and consistency of the calculated results. In addition, we have developed a method for compensating for possible slice shifts in the SA volumes that are acquired with two separate breath-holds.

\section{Method}

\subsection{MRI Data}

Fifteen chronic infarction patients who were referred for MR prior to catheter ablation of ventricular arrhythmia were involved in this study. MR imaging was performed using a 1.5T Gyroscan ACS-NT MRI scanner (Philips Medical Systems, Best, The Netherlands). After obtaining the scout and cine views, a LookLocker sequence was acquired approximately $15 \mathrm{~min}$ after bolus injection of gadolinium DPTA $(0.15 \mathrm{mmol} / \mathrm{kg})$. $\mathrm{T}_{1}$-weighted LGE images were acquired with an inversion-recovery three-dimensional turbo-field echo sequence with parallel imaging. The heart was imaged with 20 to 24 imaging levels in the SA view, and 5-10 levels in the $2 \mathrm{CH}$ and $4 \mathrm{CH}$ view. All views were acquired at the same late diastolic phase. Due to the high number of slices in the SA view, it was typically acquired as two stacks in two separate breath-holds. For the other two views, images were acquired in one stack within a single breath-hold. For the $\mathrm{SA}$ acquisition, the slice thickness is $10 \mathrm{~mm}$ with $5 \mathrm{~mm}$ overlap, with in-plane resolution of $1.56 \times 1.56 \mathrm{~mm}$; for the other two acquisitions, the slice thickness is $12 \mathrm{~mm}$ with $6 \mathrm{~mm}$ overlap, with in-plane resolution of $1.56 \times 1.56 \mathrm{~mm}$.

\subsection{Heart Registration}

Since each acquisition is performed with a separate breath-hold, the position of the heart may vary in each of the volumes. In particular, this may result in a large discontinuity in the slice direction in the SA volumes that are acquired using two breath-holds.

Registration schemes based on the image gradient, including the one used in this work, are proven to be more robust to the intensity variation in the MR data than those operating on the intensity directly. For alignment of the heart in different images, we used the FFT-based scale invariant image registration with gradient correlation [7, which allows immediate recovery of the unknown translation, rotation, and scaling between two image stacks. This method can also estimate sub-pixel image displacements, but, unfortunately, this methodology was not yet extended to $3 \mathrm{D}$.

Before performing the registration, all the image stacks have to be transformed into a common coordinate system. We achieve it by bringing all three image stacks into the coordinate space of the SA view. At this stage, we aim at rough (with voxel precision) compensation of the inter-scan heart displacement. Thus, 


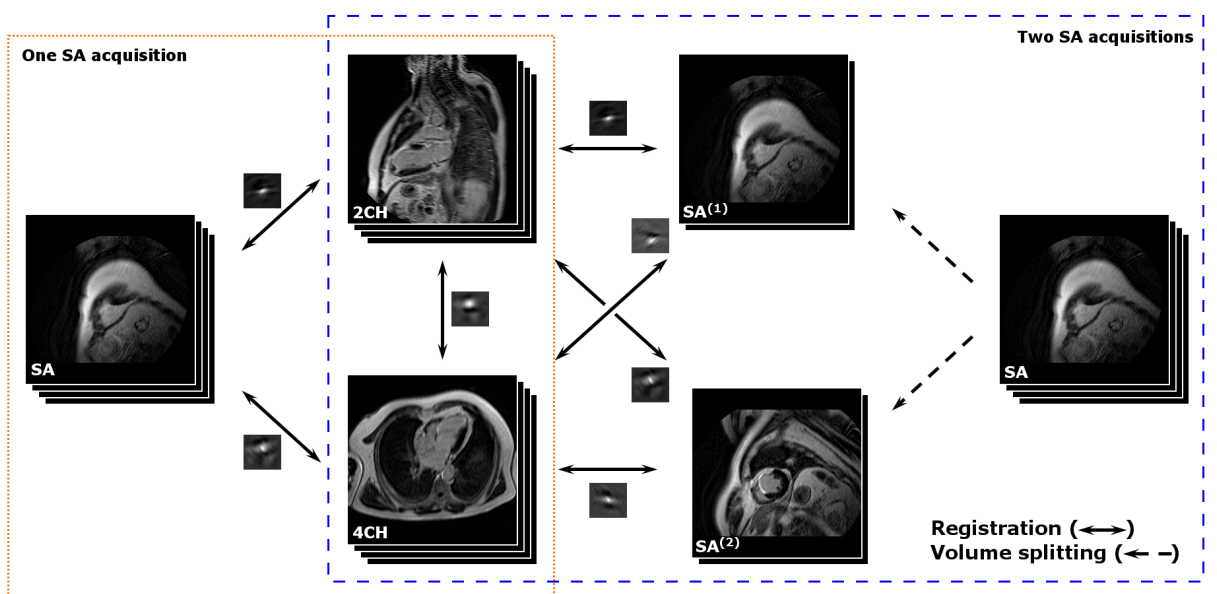

Fig. 1. Joint gradient-correlation-based registration of three orthogonal heart views

we restrict ourselves to estimation of the unknown displacement. As the region around the heart is the volume of interest, the registration method is expected to perform reliable alignment inside it while neglecting possible misalignment outside. To constrain the registration, we calculate the region where all three views intersect. Then, we find the largest right rectangular parallelepiped $R$ that fits entirely in the described overlap region. This step is necessary for performing the correlation, which expects rectangular images as input. Finally, each of the views is cropped to the region $R$.

The gradient of each view in the region $R$ is used to estimate the unknown displacements $\mathbf{d}_{\left(V_{1}, V_{2}\right)}$ between each volume pair $V_{1}, V_{2} \in\{\mathrm{SA}, 2 \mathrm{CH}, 4 \mathrm{CH}\}$. The best triple of the displacement vectors $\mathcal{D}=\left\{\mathbf{d}_{(\mathrm{SA}, 2 \mathrm{CH})} ; \mathbf{d}_{(\mathrm{SA}, 4 \mathrm{CH})} ; \mathbf{d}_{(2 \mathrm{CH}, 4 \mathrm{CH})}\right\}$ should maximize each of corresponding gradient-correlation maps

$$
\mathbf{d}_{\left(V_{1}, V_{2}\right)}=\underset{\mathbf{x}}{\arg \max } G C\left(\mathbf{x} ; V_{1}, V_{2}\right),
$$

and satisfy the vector addition rule

$$
\mathbf{d}_{(\mathrm{SA}, 2 \mathrm{CH})}+\mathbf{d}_{(2 \mathrm{CH}, 4 \mathrm{CH})}=\mathbf{d}_{(\mathrm{SA}, 4 \mathrm{CH})} .
$$

The gradient-correlation maps are scaled beforehand by dividing them by their largest value. Consequently, we calculate the set $\mathcal{D}$ by jointly analyzing all three correlation maps (see left panel of the Fig. 1) for all possible combinations of displacements. In order to limit the search space, for each correlation map we select only the points with the intensity higher than 99.99 percentile of all the intensity values.

\subsection{Slice Shift Correction in SA Volume}

For correction of a possible slice shift in the SA view, we developed a method that utilizes information from the other two views. More precisely, we split the 
entire $\mathrm{SA}$ volume into two sub-volumes $\mathrm{SA}^{(1)}$ and $\mathrm{SA}^{(2)}$, each acquired within a separate breath-hold. Consequently, both sub-volumes are registered to the $2 \mathrm{CH}$ and the $4 \mathrm{CH}$ volumes. This procedure, illustrated in the right panel of Fig. 1, is similar to the one described above. The only difference is that in this case joint estimation of the displacements is performed on five gradient correlation maps

$$
\mathcal{D}=\left\{\mathbf{d}_{\left(\mathrm{SA}^{(1)}, 2 \mathrm{CH}\right)}, \mathbf{d}_{\left(\mathrm{SA}^{(1)}, 4 \mathrm{CH}\right)}, \mathbf{d}_{\left(\mathrm{SA}^{(2)}, 2 \mathrm{CH}\right)}, \mathbf{d}_{\left(\mathrm{SA}^{(2)}, 4 \mathrm{CH}\right)} ; \mathbf{d}_{(2 \mathrm{CH}, 4 \mathrm{CH})}\right\}
$$

with the following constraints

$$
d_{\left(S A^{k}, 2 C H\right)}+d_{2 C H, 4 C H)}=d_{\left(S A^{k}, 4 C H\right)}, \quad k=1,2 .
$$

The unknown shift $\mathbf{d}_{\left(\mathrm{SA}^{(1)}, \mathrm{SA}^{(2)}\right)}$ between the $\mathrm{SA}^{(1)}$ and the $\mathrm{SA}^{(2)}$ is calculated based on the estimated displacements using the vector addition rule

$$
\mathbf{d}_{\left(\mathrm{SA}^{(1)}, \mathrm{SA}^{(2)}\right)}=\mathbf{d}_{\left(\mathrm{SA}^{(1)}, 2 \mathrm{CH}\right)}-\mathbf{d}_{\left(\mathrm{SA}^{(2)}, 2 \mathrm{CH}\right)}=\mathbf{d}_{\left(\mathrm{SA}^{(1)}, 4 \mathrm{CH}\right)}-\mathbf{d}_{\left(\mathrm{SA}^{(2)}, 4 \mathrm{CH}\right)} .
$$

Allowing arbitrary slice shifts may result in an unwanted gap in the image stack in case the estimated displacement $\mathbf{d}_{\left(\mathrm{SA}^{(1)}, \mathrm{SA}^{(2)}\right)}$ has a positive component in the slice direction $z$. To avoid such scenarios, we assume that the slice shift is in-plane. Mathematically this means that $d_{\left(\mathrm{SA}^{(1)}, \mathrm{SA}^{(2)}\right), z}=0$, which adds two extra constraints

$$
d_{\left(\mathrm{SA}^{(1)}, 2 \mathrm{CH}\right), z}=d_{\left(\mathrm{SA}^{(2)}, 2 \mathrm{CH}\right), z}, \quad d_{\left(\mathrm{SA}^{(1)}, 4 \mathrm{CH}\right), z}=d_{\left(\mathrm{SA}^{(2)}, 4 \mathrm{CH}\right), z} .
$$

After the slice shift $\mathbf{d}_{\left(\mathrm{SA}^{(1)}, \mathrm{SA}^{(2)}\right)}$ is calculated, the corresponding displacement is applied to $\mathrm{SA}^{(2)}$ and both parts are recombined into a single $\mathrm{SA}$ volume.

\subsection{Orthogonal Super-Resolution Reconstruction}

For calculating the high-resolution image, we use the method of Poot et al. 8]. Intensities of all three views were normalized by histogram equalization beforehand. The SSR was implemented with total variation smoothing. During reconstruction, all the affine transformation parameters: translation, rotation, and scale, are iteratively updated to allow sub-voxel precision of image alignment.

\section{Experiments and Results}

\subsection{Myocardial Scar Characterization}

SA LGE sequence is conventionally used in clinical studies to assess myocardial scar. To evaluate the improvement of the proposed SRR over SA, we interpolated the original SA volume to the same isotropic resolution as SRR by cubic spline and used it as a reference in all experiments. From both SRR and SA volume, we performed myocardial scar characterization using a validated scar segmentation method 9]. The myocardial scar region was identified using the Otsu method to differentiate normal and scar tissue. Within this region, the scar core zone and gray zone were further differentiated by the full-width-half-maximum method as validated on histological data [10]. It is worth mentioning that the scar was not necessarily at the same location among subjects. 

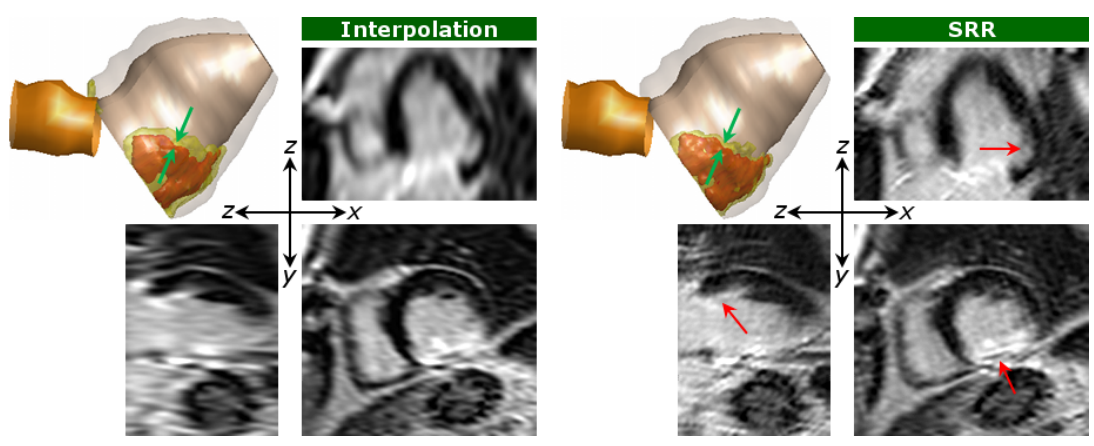

Fig. 2. Comparison between the interpolated SA and the SRR volumes. A 3D reconstruction of the myocardial scar and one slice of each orthogonal view are shown. The locations of improvement in scar definition are indicated by the red and green arrows.

\subsection{SRR Reconstruction Results}

Compared to the interpolated SA volume, similar core zone size (percent to left ventricle volume) was identified from the SRR volume: $12.0 \pm 9.2 \%$ vs $11.6 \pm$ $8.7 \%, p=N S$ (absolute size: $17.2 \pm 16.7 \mathrm{~cm}^{3}$ vs. $16.5 \pm 15.6 \mathrm{~cm}^{3}, p=N S$ ) by the nonparametric Kolmogorov-Smirnov test, but reduced gray zone size was identified: $6.2 \pm 4.7 \%$ vs $9.8 \pm 6.5 \%, p<0.05$ (absolute size: $8.4 \pm 5.2 \mathrm{~cm}^{3}$ vs. $13.2 \pm 8.0 \mathrm{~cm}^{3}, p<0.05$ ). The results were in line with previous observation that low imaging resolution does not significantly influence the scar core zone estimation, but may lead to over-estimation of scar gray zone, since PVE is pronounced in the latter zone [3]. Fig. 2] a illustrates such phenomenon in 3D, where the reduction of gray zone is obvious in the through-plane direction. The increase of image resolution is further observed in all three orthogonal views, especially in the $\mathrm{z}-\mathrm{x}$ and $\mathrm{y}-\mathrm{z}$ plane (see red arrows in Fig. 2 b).

In the interpolated SA volume, the image gradients were anisotropic (Fig. 3 b). Subtle difference was further observed between the two in-plane gradients in the SA volume. The $x$-direction is smoother than the $y$-direction, as can be attributed to the non-symmetric acquisition matrix $(256 \times 104)$ during MR image acquisition: more frequency components were encoded in the $y$-direction than in the $x$-direction. In the SRR reconstructed volume, the gradient was more homogenous in all directions as a result of integrating two orthogonal views.

\subsection{Electroanatomical Mapping}

All patients underwent catheter ablation of ventricular arrhythmias. During the procedure, high-density electroanatomical voltage mapping (EAVM) was performed on the endocardial surface to measure the electrophysiological (EP) characteristics in and around the myocardial scar region. The amplitude of the bipolar voltage reflects the local EP activities in vivo. Previous clinical studies have validated the empirical voltage thresholds for myocardial tissue characterization: normal $v>1.5 \mathrm{mV}$, scar gray zone $0.5 \mathrm{mV}<v<1.5 \mathrm{mV}$, and scar 

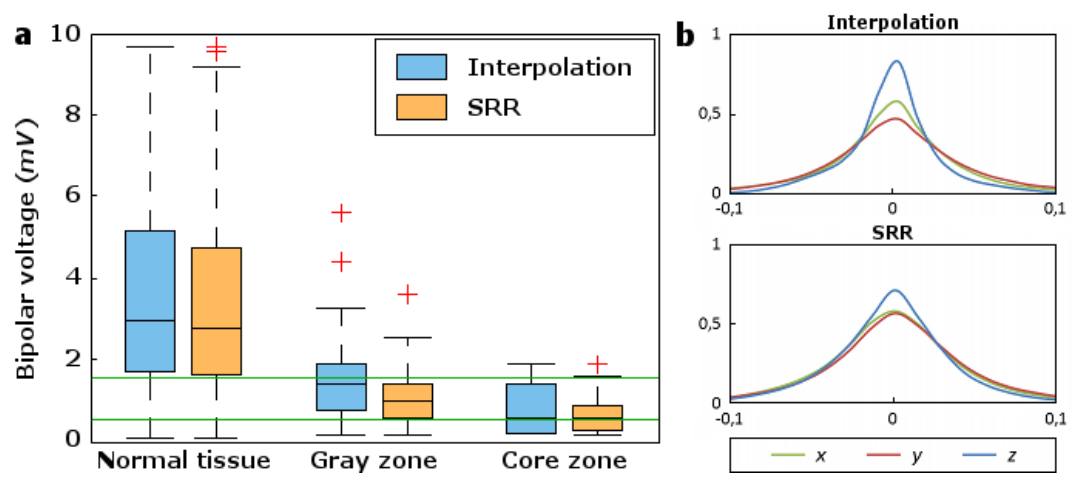

Fig. 3. Distribution of the bipolar voltages in identified normal tissue, scar gray zone, and core zone, from the interpolated SA and SRR volume (a). Green lines indicate $0.5 \mathrm{mV}$ and $1.5 \mathrm{mV}$ respectively, while the gray zone is supposed to have voltages between these two empirical thresholds. Distribution of image gradient in three directions (b).

core zone $v<0.5 \mathrm{mV}$. In absence of histological data, the EAVM measurement provides the best available gold standard for characterizing the underlying myocardial tissue composition. Registration between the EAVM and MRI data was performed by minimizing the point-to-surface distance under the assumption of a fixed heart orientation [11. To evaluate the myocardial scar characterization from the SRR and the SA volumes, the acquired bipolar voltage was labeled according to their identified location on the endocardial surface. Fig. 3 a shows the box plot of the voltages in three regions: normal myocardium, scar gray zone, and scar core zone.

No significant difference was observed between the bipolar voltages in the normal tissue and scar core zone identified from the two volumes. The difference was however pronounced at the gray zone: the bipolar voltages from the gray zones of the interpolated SA volume were higher from those from the SRR reconstructed volume, $2.14 \pm 1.38 \mathrm{mV}$ vs $1.07 \pm 0.66 \mathrm{mV}$ with $p<0.05$ by the nonparametric Kolmogorov-Smirnov test. This indicates that with the conventional SA volume, overestimation of scar gray zone can indeed occur due to PVE. In comparison, the identified gray zone from the SRR volume showed better agreement with clinical EAVM value (between the two horizontal lines of EAVM voltage), indicating improved gray zone characterization.

\section{Conclusion}

By integrating image information from the $\mathrm{SA}$, the $2 \mathrm{CH}$, and the $4 \mathrm{CH} \mathrm{LGE}$ sequences, we are able to reconstruct a high-resolution myocardial scar representation. Our method uses the SRR framework to reconstruct the isotropic volume from the three anisotropic volumes. For compensation of the inter-scan motion of the heart, whose precise alignment is a crucial prerequisite for success 
of the SRR reconstruction, we have developed a method that jointly estimates the optimal and consistent positioning of the volumes with respect of each other. An important property of this method is that it is very well suited for the MR data as it is based on the gradient information. The proposed method enables more accurate characterization of myocardial scar compared to conventional SA LGE volume, especially with respect to the clinically significant gray zone, allowing improved risk profiling and procedure planning in individual patients.

\section{References}

1. Yan, A.T., Shayne, A., Brown, K.A., Gupta, S., Chan, C., Luu, T., Carli, M.D., Reynolds, H.G., Stevenson, W.G., Kwong, R.Y.: Characterization of the periinfarct zone by contrast-enhanced cardiac magnetic resonance imaging is a powerful predictor of post-myocardial infarction mortality. Circulation 114, 32-39 (2006)

2. Kim, R.J., Fieno, D.S., Parrish, T.B., Harris, K., Chen, E.L., Simonetti, O., Bundy, J., Finn, J.P., Klocke, F.J., Judd, R.M.: Relationship of MRI delayed contrast enhancement to irreversible injury, infarct age, and contractile function. Circulation 100(19), 1992-2002 (1999)

3. Schelbert, E.B., Hsu, L.Y.Y., Anderson, S.A., Mohanty, B.D., Karim, S.M., Kellman, P., Aletras, A.H., Arai, A.E.: Late gadolinium-enhancement cardiac magnetic resonance identifies postinfarction myocardial fibrosis and the border zone at the near cellular level in ex vivo rat heart. Circulation. Cardiovascular Imaging 3(6), 743-752 (2010)

4. Goetti, R., Kozerke, S., Donati, O.F., Sürder, D., Stolzmann, P., Kaufmann, P.A., Lüscher, T.F., Corti, R., Manka, R.: Acute, subacute, and chronic myocardial infarction: Quantitative comparison of $2 \mathrm{D}$ and $3 \mathrm{D}$ late gadolinium enhancement $\mathrm{MR}$ imaging. Radiology 259, 704-711 (2011)

5. Shilling, R.Z., Robbie, T.Q., Bailloeul, T., Mewes, K., Mersereau, R.M., Brummer, M.E.: A super-resolution framework for 3-D high-resolution and high-contrast imaging using 2-D multislice MRI. IEEE Trans. Med. Imag. 28(5), 633-644 (2009)

6. Ur Rahman, S., Wesarg, S.: Upsampling of cardiac MR images: Comparison of averaging and super-resolution for the combination of multiple views. In: ITAB 2010, University of Ioannina, pp. 1-4. IEEE Inc., New York (2010)

7. Tzimiropoulos, G., Argyriou, V., Zafeiriou, S., Stathaki, T.: Robust FFT-based scale-invariant image registration with image gradients. IEEE Trans. Pattern Anal. Mach. Intell. 32, 1899-1906 (2010)

8. Poot, D.H.J., Van Meir, V., Sijbers, J.: General and efficient super-resolution method for multi-slice MRI. In: Jiang, T., Navab, N., Pluim, J.P.W., Viergever, M.A. (eds.) MICCAI 2010, Part I. LNCS, vol. 6361, pp. 615-622. Springer, Heidelberg (2010)

9. Tao, Q., Milles, J., Zeppenfeld, K., Lamb, H., Bax, J., Reiber, J., van der Geest, R.J.: Automated segmentation of myocardial scar in late enhancement MRI using combined intensity and spatial information. Magn. Reson. Med. 64, 586-594 (2010)

10. Amado, L., Gerber, B., Gupta, S., Rettmann, D., Szarf, G., Schock, R., Nasir, K., Kraitchman, D., Lima, J.: Accurate and objective infarct sizing by contrastenhanced magnetic resonance imaging in a canine myocardial infarction model. J. Am. Coll. Cardiol. 12, 2383-2389 (2004)

11. Tao, Q., Milles, J., van Huls van Taxis, C., Lamb, H., Bax, J., Reiber, J., Zeppenfeld, K., van der Geest, R.J.: Toward magnetic resonance-guided electroanatomical voltage mapping for catheter ablation of scar-related ventricular tachycardia: A comparison of registration methods. J. Cardiovasc. Electr. 23, 74-80 (2012) 